\section{SYMBOL ERROR PROBABILITY OF 16-QAM SYSTEM OVER AWGN AND RAYLEIGH FADING CHANNELS}

'Oyetola, Oluwadamilola Kehinde; 'Okubanjo, Ayodeji Akinsoji; 'Okandeji, Alexander Akpofure; Alao, Peter Olufemi; ${ }^{\mathrm{a} O}$ Osifeko, Martins $\mathrm{O}$. and ${ }^{\mathrm{a} O}$ Olasunkanmi, Omowunmi Grace ${ }^{a}$ Department of Computer and Electrical/Electronic Engineering,

Faculty of Engineering, Olabisi Onabanjo University, Ibogun Campus, Ogun, Nigeria.

${ }^{b}$ Department of Electrical and Electronic Engineering, Unversity of Lagos, Akoka

*Corresponding author's e-mail:oyetola.oluwadamilola@oouagoiwoye.edu.ng

okubanjo.ayodeji@oouagoiwoye.edu.ng:

\section{ABSTRACT}

Wireless communication had transformed the mode of human interactions in recent times, distance is no longer a barrier as messages can be sent several miles apart within few seconds. In addition, the pervasive adoption of mobile communication system had engendered researchers to device new and obvious in the deployment of trending mobile generations such as $2 \mathrm{G}, 3 \mathrm{G}$ and $4 \mathrm{G}$ systems for high speed voice and data services. Nevertheless, these systems are still embattled with unpredictable impairment such as noise in fading channels that impedes optimal system performance. In this paper, performance evaluation of 16-Quadrature Amplitude Modulation (16-QAM) system over Additive White Gaussian Noise (AWGN) and Rayleigh Channels using simulated and theoretical approach is presented. Theoretical mathematical expression for Symbol Error Rate(SEP) was derived, an simulation was setup using MATLAB/SIMULINK for performance evaluation. The results show that SEP is dependent on signal-noise-ratio (SNR) for both methods. However, SEP wasvery high fo Rayleigh channel as compared with AWGN.

Keywords: Symbol error probability, Quadrature amplitude modulation, Rayleigh fading, wireless communication, noise

Accepted Date: $15^{\text {th }}$ March 2018

\section{INTRODUCTION}

The evolution of wireless communication in the last two decades had facilitated widespread adoption of wireless systems for effective means of disseminating information to one another on real time bases irrespective of distance. (Essop \& Xu, 2015). Currently, many smart phones and mobile devices have up to eight radios optimized for receiving signals from different frequency bands, such as WiFi (2.4GHz), LTE (Long Time Evolution, $800 \mathrm{MHz}$ ), GSM (Global System for Mobile Communication, 900MHz), Bluetooth $(2.4 \mathrm{GHz}), \mathrm{FM}$ radio $(100 \mathrm{MHz})$ and many more (Stewart, Barlee, Atkinson, \& Crockett, 2015). However, certain unavoidable circumstances deter these systems from achieving optimum distribution is adopted to describe the statistical fluctuations of signals received from a multipath

performance. Inherently, a communication system consist of a transmitter, a channel and a receiver. The radio channel between the transmitter and receiver varies from line-of-sight to multipath fading channel due to obstruction from building, trees, mountains and other high objects. The randomness in the mobile channel is the core characteristic that make it differ from predictable stationary wired channel. In essence, wion channels suffer from impairments such as noise, attenuation, distortion, fading and interference that often results into receiving signals with errors (Proakis \& Salehi, 2008). However, the characteristics of a communication channel determine which impairments apply to that particular channel and which are the determining factors in the performance of the channel.

Noise is present in all communication channels and it is the major impairment in many communication systems, so the effect of noise on the reliability and performance of modulation systems cannot be ignored. It is therefore pertinent to investigate the isolated effect of noise on communication system using a simple model of noise such as Additive White Gaussian noise (AWGN) channel model to achieve the fundamental understanding of noise effects on digital systems. Similarly, Rayleigh fading con fading chan of probability of quadr WWGN and fation (QAM) system over AWGN and fading channels such as Rayleigh fading channel were examine using different modeling approaches. In the paper (Kim, Kim, Jeong, Mun, \& Lee, 1996), the authors derived the symbol error probability for QAM with L- fold space diversity in Rayleigh fading channels. They proposed two combining techniques, maximal ratio combining (MRC) and selection combining (SC), which overcomes the limitations of deriving the symbol entre rete (SER) of QAM with two branch MRC space diverity. Analyical resuls showed the the probaility of eror decreses with the order of diversity, and the increm dectal the gain per additional branch decreases as the number of branches becomes larger. Hua Yu and co-worker in their paper (Yu \& Wei, 2010), derived the exact SEP performance of cross QAM in Rayleigh flat fading channels. The closed-form SEP expression obtained is simple and details only basic functions. their simulation produced results similar to analytical results.

increase in the error rate. This paper present an indepth analysis of 16-QAM system over AWGN an Rayleigh channels using simulation and theoretical approach. QAM modulation technique is a two dimensional modulation technique and it requires two orthonormal basis functions which allows two different signals to be sent simultaneously on the came che the anplitude is allowed to vary with the phase. Interestingly, QAM is a digital communication modulation technique extensively used in moder wireless communication system, such as WiMax, LTE, WCDMA, HSDPA/HSUPA, etc.(Ezea, Adebusuyi, Ofusori, \& Ezea, 2018; Ndujiuba, Oni, \& Ibhaze, 2015) and investigating its performance is sequential to achieving improved voice and dat services in contemporary communication systems.

\section{Mathematical Model of QAM system}

Quadrature Amplitude Modulation is a popula system of attaining high data rates in bandwidth channels that are limited. It is characterized by two data signals that are $90^{\circ}$ out of phase with each other. M-ary QAM has become dominant over the years due to the high spectral efficiency . For a QAM system, the transmitted signal can be expressed as follows:

$s_{m i}(t)=\operatorname{Re}\left[\left(A_{m i}+j A_{m q}\right) g(t) e^{j 2 \pi f t}\right]=$

$A_{m i} g(t) \cos 2 \pi f t-A_{m q} g(t) \sin 2 \pi f t$

where $A_{m i}$ and $A_{m q}$ are the information bearing signal amplitudes of the quadrature carriers and $g(t)$ is the signal pulse shape.

In vector representation,

In addition, (Chy \& Khaliluzzaman, 2015), investigated the evaluation of signal-to-noise ratio (SNR) in terms of constant bit error rate on AWGN, Rayleigh and Rician fading channels. The simulation showed that, Rician shows better performance as compared to AWGN and Rayleigh. (Omijeh \& Eyo, 2016) performed a compalive (Omijen \& Eyo, 2016) perforned a conparative M-PSK, and M-QAM) $(\mathrm{M}=2,4,8,16,32$, and 64$)$ M-PSK, and $\mathrm{M}-\mathrm{QAM})(\mathrm{M}=2,4,8,16,32$, and 64)
Modulation Techniques in AWGN Channel. The analysis of the graphical illustration of $\mathrm{Eb} / \mathrm{No}$ vs BER of these M- PSK schemes showed that increase in the value of $M$ causes a corresponding

$$
\begin{aligned}
s_{m} & =\left(s_{m i}+s_{m q}\right) \\
& =\left(A_{m i} \sqrt{\frac{E_{g}}{2}}, A_{m \varphi} \sqrt{\frac{E_{g}}{2}}\right)
\end{aligned}
$$

where $E_{\mathrm{g}}=$ energy content of signal $g(t)$ for bandpass signal. 


$$
E_{m}=\left\|S_{m}\right\|^{2}=\frac{E_{g}}{2}\left(A_{m i}{ }^{2}+A_{m q}{ }^{2}\right)
$$

For equiprobable signal $P_{e}=\frac{1}{M} \sum_{m}^{M} P\left[\right.$ error $\left.\left.\right|_{m}\right]$

where $E_{m}$ is the energy content of $s_{m}(t)$
The Euclidean distance between any pair of signa

where $E_{m}$ is the energy content of $s_{m}(t)$ vector in QAM is

$$
\begin{aligned}
& d_{m n}=\sqrt{\mid s_{m}-s_{n} \|^{2}} \\
& =\sqrt{\frac{E_{g}}{2}\left[\left(A_{m i}-A_{n i}\right)^{2}+\left(A_{m q}-A_{m q}\right)^{2}\right]},
\end{aligned}
$$

where $S_{m}$ and $S_{n}$ are the signal vectors at point $m$ and $n$, respectively:

For a case of rectangular constellation as shown in Fig 1, the minimum distance $\left(d_{\min }\right)$ between adjacent points

$d_{\min }=\sqrt{\frac{6 \log _{2} M}{(M-1)} E_{b a v g}}$

(Proakis \& Salehi, 2008)

$E_{b a v g}=$ average signal energy.

\section{Error Probability of QAM System}

Using rectangular constellation,

$M-$ ary $\mathrm{QAM}=2 \sqrt{M}-$ ary $\mathrm{PAM}$

The probabilities of correct decision in QAM is the product of correct decision of the two PAM systems

i.e. $P_{c M-Q A M}=P_{c \sqrt{M}-P A M}^{2}=\left(1-P_{e \sqrt{M}-P A M}\right)^{2}$

Where $P_{C M-O A M}=$ probability of correct decision for QAM

$P_{c \sqrt{M}-P A M}=$ probability of correct decision for PAM $P_{e \sqrt{M}-P A M}=$ Symbol error probability (SEP) or probability of wrong decision for PAM

$\therefore P_{e M-Q A M}=1-\left(1-P_{e \cdot M-P A M}\right)^{2}=$

$2 P_{e 、 M-P A M}\left(1-\frac{1}{2} P_{e, M-P A M}\right)$

$P_{e M-Q A M}=$ Symbol error probability (SEP) for QAM

But

$P_{e}=\sum_{m}^{M} p_{m} P\left[\right.$ error $\left.\left.\right|_{m}\right]=$ symbol error probability.
In PAM systems there are (M-2) inner point and 2 outer points in the constellation. Therefore there two forms of errors as thus:

$P_{e i n}=P\left[n>\frac{1}{2} d \mathrm{~min}\right]$ and $P_{e o n t}=\frac{1}{2} P_{e i n}$

for inner and outer point respectively

Hence $P_{e M-P A M}=\frac{1}{M}\left[(M-1) P_{e i n}+2 P_{e o u t}\right]$

\section{AWGN Channel Model}

Additive White Gaussian Noise channel can be model using standard normal random variable, having a probability density function:

$f_{X}(x)=\frac{1}{\sqrt{2 \pi}} e^{-x^{2}: 2}$

Using (8) and noise variable $\mathrm{n}$ with zero mean and variance

$\sigma^{2}=\frac{N_{0}}{2}$, where $N_{0}$ is the noise component

$\Rightarrow P_{e i n}=\frac{1}{\sqrt{\pi N_{0}}} \int_{-\infty}^{x} e^{-} \frac{\left(d_{\min } / 2\right)^{2}}{N_{0}} d n$

where $d n=$ distance of noise variable

Let $\quad x=\frac{-d_{\min }}{\sqrt{2 N_{0}}}, x=\frac{-d\left(d_{\min }\right)}{\sqrt{2 N_{0}}}=d x$,

$d n=d\left(d_{\min }\right)=-\sqrt{2 N_{0}} d x$

By changing boundary using $(9)$

$P_{\text {ein }}=\frac{-2}{\sqrt{2}} \int_{-\infty}^{\substack{d_{\min } \\-2 N_{0}}} e^{-} \frac{x^{2}}{2} d x=\frac{2}{\sqrt{2}} \int_{\frac{d_{\min }}{\sqrt{2} 2 N_{0}}}^{\infty} e^{-} \frac{x^{2}}{2} d x$

$P_{\text {ein }}=2 Q\left(\frac{d_{\min }}{\sqrt{2 N_{0}}}\right), P_{\text {eout }}=P_{\text {ein }}=Q\left(\begin{array}{l}d_{\min } \\ 2 N_{0}\end{array}\right)$

Using (10) in (7)

$$
\begin{aligned}
& P_{e, M-P, M}={ }_{M}\left[2(M-1) Q\left(\frac{d_{\min }}{2 N_{0}}\right)+2 Q\left(\frac{d_{\min }}{\sqrt{2 N_{0}}}\right)\right] \\
&=2\left(1-\frac{1}{M}\right) Q\left(\frac{d_{\min }}{\sqrt[2]{2 N_{0}}}\right) \\
& \text { Hence } \quad P_{Q, M-P A M}=2\left(1-\frac{1}{\sqrt{ } M}\right) Q\left(\frac{d_{\min }}{2 N_{0}}\right) \quad(11)
\end{aligned}
$$

Putting (11) into (5)

$$
P_{e M-Q A M}=4\left(1-\frac{1}{\sqrt{M}}\right) Q\left(\frac{d_{\min }}{\sqrt{2 N_{0}}}\right)\left[1-\left(1-\frac{1}{\sqrt{M}}\right) Q\left(\frac{d_{\min }}{\sqrt{2 N_{0}}}\right)\right]
$$

Substituting (3) into (12)

$$
P_{e M-Q A M}=4\left(1-\frac{1}{\sqrt{M}}\right) Q\left(\sqrt{\frac{3 \log _{2} M}{(M-1)} \frac{E_{\text {bavg }}}{N_{0}}}\right)-4\left(1-\frac{1}{\sqrt{M}}\right)^{2} Q^{2}\left(\sqrt{\frac{3 \log _{2} M}{(M-1)} \frac{E_{\text {bavg }}}{N_{0}}}\right) \text { (13) }
$$

For 16 QAM, M=16, (16) becomes

$$
\begin{aligned}
& P_{\text {e16-QAM_AWGN }}=3 Q\left(\sqrt{\frac{4}{5} \frac{E_{\text {bavg }}}{N_{0}}}\right)-\frac{9}{4} Q^{2}\left(\sqrt{\frac{4}{5} \frac{E_{\text {bavg }}}{N_{0}}}\right) \\
& \text { But } \quad Q(x)=\frac{1}{2} \operatorname{erfc}\left(\frac{x}{\sqrt{2}}\right) \\
& \text { Hence, } \quad P_{\text {el6-QAM_AWGN }}=1.5 \operatorname{erfc}\left(\sqrt{0.4 \frac{E_{\text {bavg }}}{N_{0}}}\right)-1.125 \operatorname{erfc}^{2}\left(\sqrt{0.4 \frac{E_{\text {bavg }}}{N_{0}}}\right)(15)
\end{aligned}
$$

(15) is the expression for theoretical Symbol Error Probability (SEP) for 16QAM in AWGN channel

\section{Rayleigh Channel Mode}

For Rayleigh channel, SEP can be expressed as follows: 


\section{$P_{R a y}=\int_{0}^{\infty} P_{e}(\gamma) \cdot P_{\gamma}(\gamma) d \gamma$}

$P_{e}(\gamma)$ is the conditional SEP, for 16-QAWGN, $P_{e}(\gamma)=P_{e 16-Q A M}{ }_{-A W G N}(\gamma)$

$P_{e 16-Q A M_{-} A W G N}(\gamma)=3 Q\left(\sqrt{\frac{4}{5} \gamma}\right)-\frac{9}{4} Q^{2}\left(\sqrt{\frac{4}{5} \gamma}\right)$

$P_{\gamma}(\gamma)=\frac{1}{\gamma_{0}} e^{-\frac{\gamma}{\gamma_{0}}}$

$\gamma$ and $\gamma_{0}$ are Signal-to-no ise ratio (SNR) and average SNR, respectively

Also $\quad Q(x)=\frac{1}{\pi} \int_{0}^{\frac{\pi}{2}} e^{-\left[\frac{x^{2}}{2 \sin ^{2} \theta}\right]} d \theta$

$Q^{2}(x)=\frac{1}{\pi} \int_{0}^{\frac{\pi}{4}} e^{\left[\frac{x^{2}}{2 \sin ^{2} \theta}\right]} d \theta$

(Marvin K. Simon, 2000)

Using (17), (19) and (20) in (16)

$P_{e 16-Q A M_{-} \text {Rayleih }}=\frac{3}{\pi} \int_{0}^{\frac{\pi}{2}} \int_{0}^{\infty} e^{-\left[\frac{0.8 \gamma}{2 \sin ^{2} \theta}\right]} P_{\gamma}(\gamma) d \gamma-\frac{9}{4 \pi} \int_{0}^{\frac{\pi}{4}} \int_{0}^{\infty} e^{-\left[\frac{0.8 \gamma}{2 \sin ^{2} \theta}\right]} P_{\gamma}(\gamma) d \gamma$

Let $\quad F_{\gamma}(s)=\int_{0}^{\infty} e^{-S_{y}} P_{\gamma}(\gamma) d \gamma \quad$ a replica of Laplace Transform

$F_{\gamma}(s)=\int_{0}^{\infty} e^{-S_{\gamma}} \frac{1}{\gamma_{0}} e^{-\frac{\gamma}{\gamma_{0}}} d \gamma$

$$
=\frac{1}{1+s \gamma_{0}}
$$

Let $s=\frac{0.8}{2 \sin ^{2} \theta}$ and comparing (22a) and (22b) with (21)

$$
\begin{aligned}
P_{\text {e16-QAM_Rayleih }} & =\frac{3}{\pi} \int_{0}^{\frac{\pi}{2}} F_{\gamma}\left(\frac{0.8 \gamma}{2 \sin ^{2} \theta}\right) d \theta-\frac{9}{4 \pi} \int_{0}^{\frac{\pi}{4}} F_{\gamma}\left(\frac{0.8 \gamma}{2 \sin ^{2} \theta}\right) d \theta \\
& =\frac{3}{\pi} \int_{0}^{\frac{\pi}{2}}\left(1+\frac{0.8 \gamma_{0}}{2 \sin ^{2} \theta}\right)^{-1} d \theta-\frac{9}{4 \pi} \int_{0}^{\frac{\pi}{4}}\left(1+\frac{0.8 \gamma_{0}}{2 \sin ^{2} \theta}\right)^{-1} d \theta \\
& =P_{\text {el6-QAM_Rayleih }}(1)-P_{\text {e16-QAM_Rayleih }}(2)
\end{aligned}
$$

$$
\begin{aligned}
P_{e 16-Q A M_{-} \text {Rayleih }}(1) & =\frac{3}{\pi} \int_{0}^{\frac{\pi}{2}}\left(1+\frac{0.8 \gamma_{0}}{2 \sin ^{2} \theta}\right)^{-1} d \theta \\
& =\frac{3}{2}-\frac{3}{\pi} \int_{0}^{\frac{\pi}{2}}\left(\frac{0.8 \gamma_{0}}{2 \sin ^{2} \theta+0.8 \gamma_{0}}\right) d \theta
\end{aligned}
$$

Let $\tan \theta=t, \frac{d t}{d \theta}=\sec ^{2} \theta=1+\tan ^{2} \theta=1+t^{2}, d \theta=\frac{1}{1+t^{2}} d t$ and $\sin \theta=\frac{t}{\sqrt{1+t^{2}}}$ (24)

Putting all the expressions in (24) in (23)

$$
\begin{aligned}
P_{e 16-Q A M_{-} \text {Rayleih }}(1) & ==\frac{3}{2}-\frac{3}{\pi} 0.8 \gamma_{0} \int_{0}^{\frac{\pi}{2}} \frac{1+t^{2}}{0.8 \gamma_{0}+\left(2+0.8 \gamma_{0}\right) t^{2}} \times \frac{1}{1+t^{2}} d t \\
& =\frac{3}{2}\left(1-\sqrt{\frac{0.8 \gamma_{0}}{2+0.8 \gamma_{0}}}\right)
\end{aligned}
$$

Using the same approach for $P_{e 16-Q A M \_ \text {Rayleih }}(2)$

$$
P_{e 16-Q_{A} M_{-} \text {Rayleih }}(2)=\frac{9}{16}\left[1-\sqrt{\frac{0.8 \gamma_{0}}{2+0.8 \gamma_{0}}}\left(\cdot \frac{4}{\pi} \tan ^{-1} \sqrt{\frac{2+0.8 \gamma_{0}}{0.8 \gamma_{0}}}\right)\right]
$$

Hence,

$$
P_{e 16-Q A M_{-} \text {Rayleih }}=\frac{3}{2}\left(1-\sqrt{\frac{0.8 \gamma_{0}}{2+0.8 \gamma_{0}}}\right)-\frac{9}{16}\left[1-\sqrt{\frac{0.8 \gamma_{0}}{2+0.8 \gamma_{0}}}\left(\cdot \frac{4}{\pi} \tan ^{-1} \sqrt{\frac{2+0.8 \gamma_{0}}{0.8 \gamma_{0}}}\right)\right]
$$

(25) is the expression for theoretical Symbol Error Probability (SEP) for 16QAM in Rayleigh channel

\section{MATLAB Simulation}

The simulation for the purpose of achieving the aim of this research is done using MATLAB / Simulink model. Function were also developed and employed to carry out the experiment. The Simulink model is shown in Fig. 2 and Fig. 3.As illustrated, random integers were generated to represent transmitted symbols which were modulated using rectangular QAM object before $t$ Rayleigh channels object models. The code Rayleigh chan the of the simulation is as follows: for $\mathrm{i}=1: 1$ :length(EbNo)

received Signal Awgn = awgn (data Mod, Eb No Lin(I), measured); s Plot Fig = scatter plot (received Signal Awgn, 1,0,'g.');

hold on

scatter plot (dataMod, 1,0,'k','sPlotFig)

(cConstellation or Transmilted Symbols for SNR = ', Signal Awgn, $\mathrm{M}, 0$, , bin'):

sym Error Ratio(i)] = symerr (data

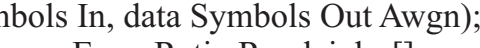

end sym Error Ratio Ray leigh= $=$;

sym Error Ratio Ray leigh= [symError Ratio Ray leigh QAM_rayleigh 2 (M, Eb No (i))]; end figure(1): 
semilogy (Eb No, symError Ratio,' xr-', 'Line width',2); hold on;

xlabel ('E_b / N_o (dB)')

ylabel ('SER');

AWGN Rayleigh Channel');

figure(1);

semilogy (EbNo, Pe sim, 'g-t','Line width',2);

$\%$ Theoretical SER

SER_16QM_AWGN $=3 *$ qfunc $(\operatorname{sqrt}(0.8 *$ EbNoLin2 $))$ -

$(9 / 4) *$ ffunc(sqrt $(0.8 * \operatorname{EbNoLin} 2))$.

func (sqrt $\left(0.8^{*} \mathrm{EbNoLin} 2\right)$

Semilogy (EbNo,SER_16QM_AWGN,'b-+','Linewidth',2) SER_16QAM_Rayleigh $=1.5 . *{ }^{*}\left(1-\operatorname{sqrt}\left(\left(0.8 . *{ }^{*}\right.\right.\right.$ EbNoLin 2$)$ (2+0.8* EbNoLin2)) $0.5625 *$ (1 -sqrt ((0.8.*EbNoLin $)$ .$(0.8 *$ EbNoLin2)) ));

16QAM_Rayleigh,'y-

grid on; legend ('AWGN Sim', 'Rayleigh Sim', 'AWGN Theory', 'Rayleigh Theory');

\section{Result and Discussion}

In order to evaluate SEP of 16-QAM system over AWGN and Rayleigh fading channels, the values of SEP with respect to ratio of bit energy to noise power spectral density $(\mathrm{Eb} / \mathrm{No})$ which is a function of SNR is noted and recorded. As shown in Fig. 6, the plot of SEP over Eb/No details the effect of SNR on performance of the system. It is observed that for both theoretical and simulation models, SEP reduces as SNR increases over the two channels. It is also germane to note that simulation results trail that of theoretical expression closely, confirming the correctness of the results. However, SEP is higher in Rayleigh channel as compared to AWGN channel. Consequently, wireless systems have poor SEP performance because of fading. This is also corroborated with the constellation plot of Fig. 4 and Fig. 5 which depicts the signal received over AWGN and Rayleigh channel at SNR value of 22.0206 respectively. The constellations reveal that information containing signal can still be detected for AWGN with simple processing which is not achievable with the same SNR in Rayleigh channel. In essence, the a over Rayleigh channel, signal power needs to be increased in the multiple of thousands of power required to transmit over wired system. This in turn incur high cost and complexity on system design and implementation.

\section{Conclusions}

This paper has thoroughly considered the performance of 16-QAM system over two fundamental channel models $\mathrm{V}$ is: AWGN and Rayligh using simul Rayleigh using simulated and Mathematical expressions were derived for SEP of 16QAM system over both channels using probability and communication theories. Also, Matlab functions were developed to model an generate symbol error rate probability (SEP) dat of each channel for performance analysis. Usin these theorical and simulat data plot of SEP plot of SE against the signal to noise ratio (SNR) wa presented. It was observed that the system has better performance over AWGN channel as compared with Rayleigh channel. The resul validated known challenges of poor performance of communication systems over wireless channels. To enjoy high throughput and better spectra efficiency, scheme such as diversity and maximum ratio combining (MCR) can be employed for multipleinput multiple-output (MIMO) systems to neutralise the effective of multipath fading.

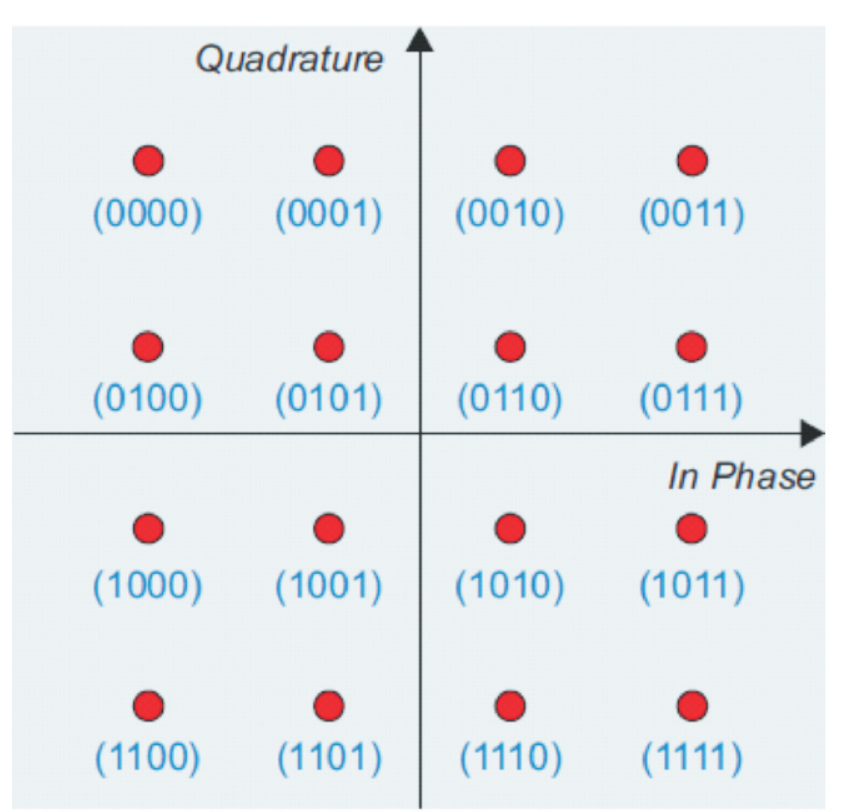

Fig. 1: 16-QAM Rectangular Constellation

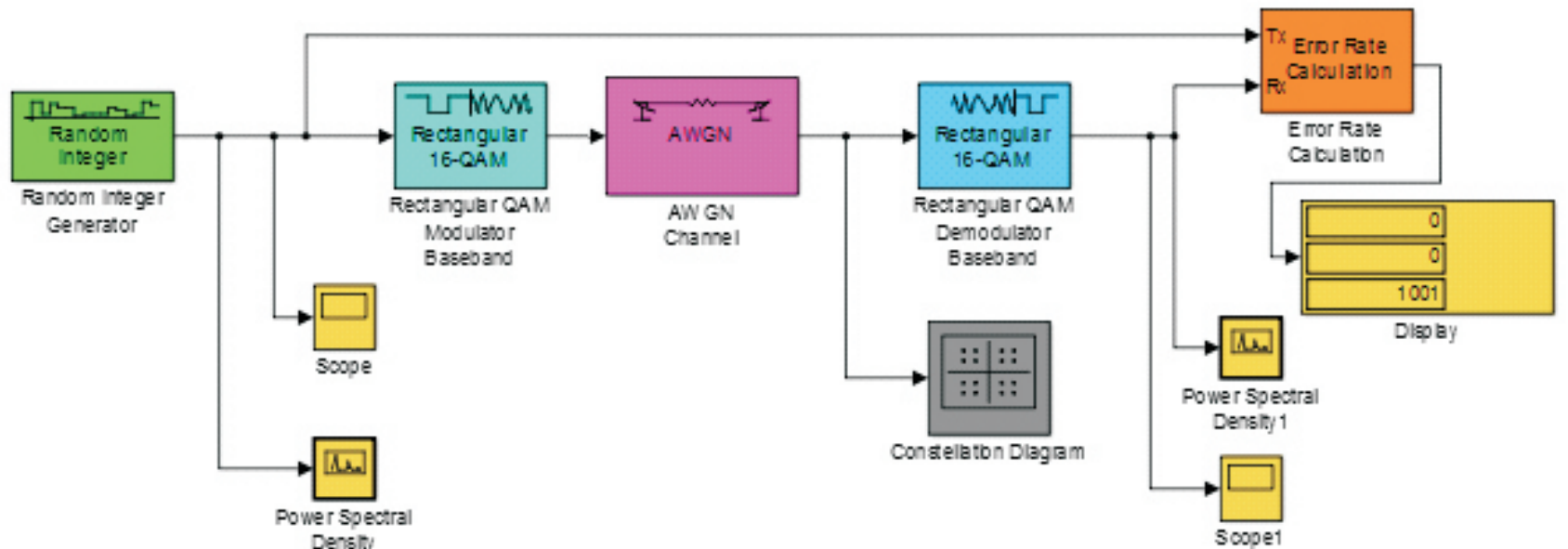

Fig. 2: Simulink Model of 16-QAM over AWGN Channel

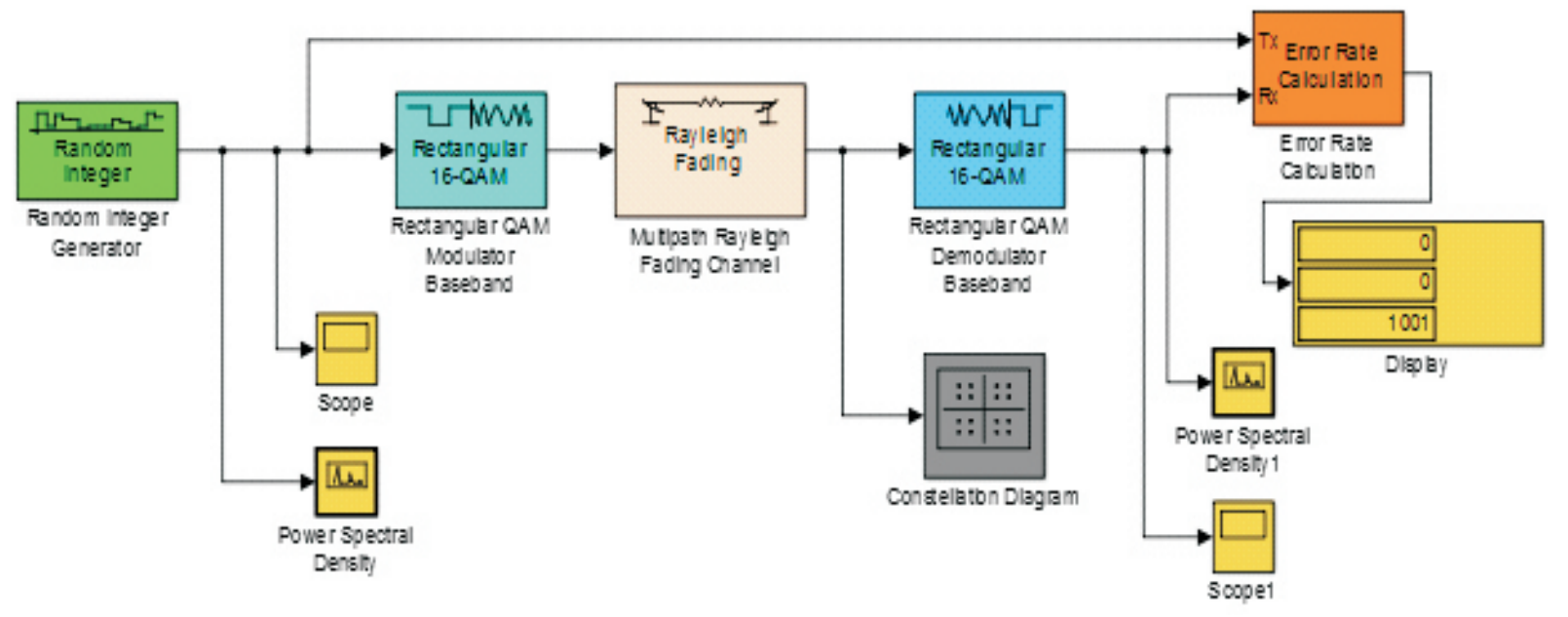

Fig. 3: Simulink Model of 16-QAM over Rayleigh Channe 
Constellation of Transmitted Symbols for SNR $=22.0206$ in AWGN channel

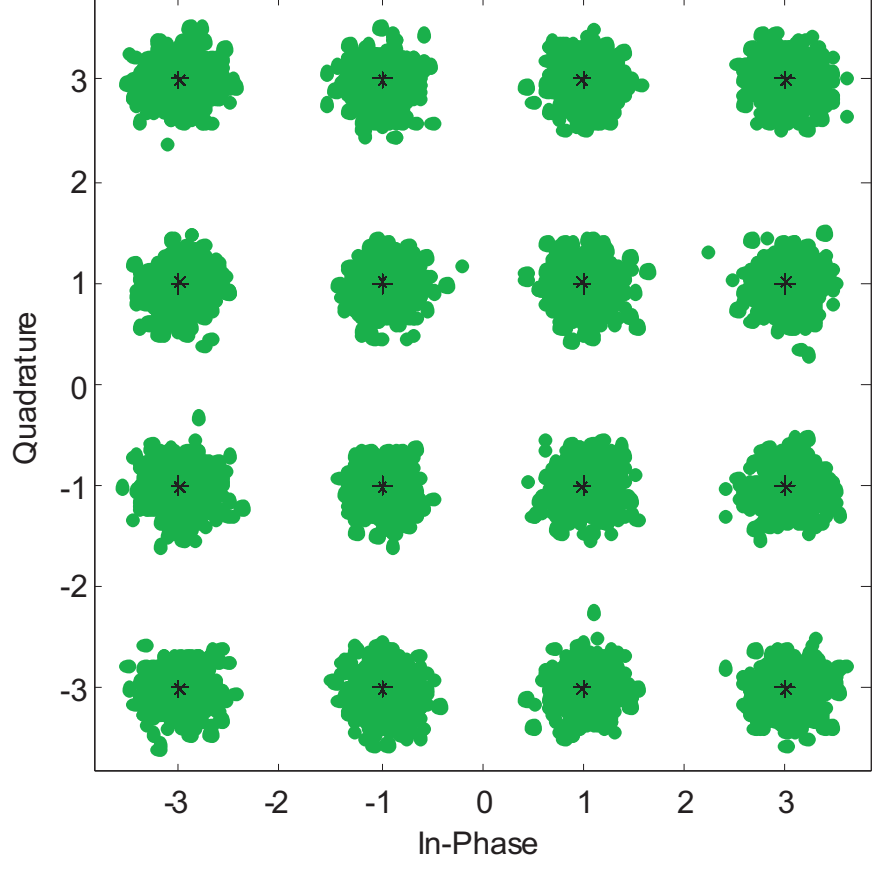

Fig. 4: Constellation of Transmitted Symbols over AWGN

Constellation of Transmitted Symbols for SNR $=22.0206$ in Rayleigh channel

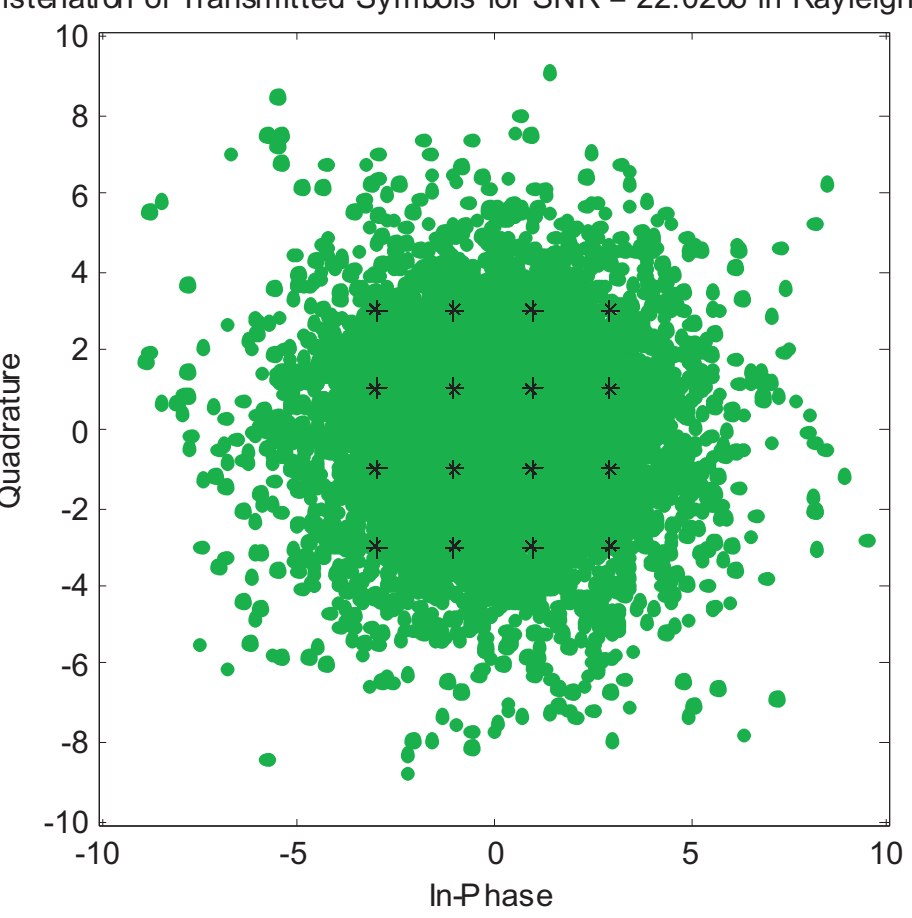

Fig.5: Constellation of Transmitted Symbols over Rayleigh

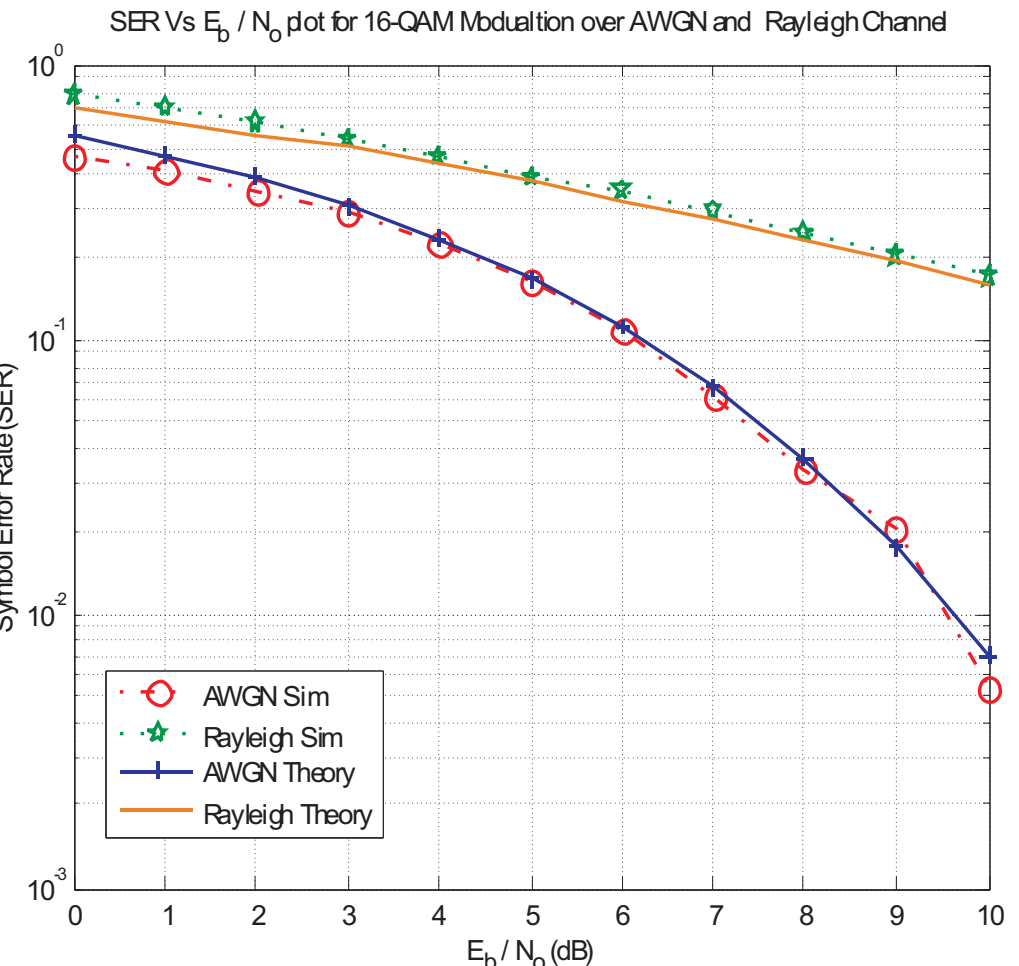

Fig. 6: SER versus Eb/No Plot

\section{REFERENCES}

Chy, D. K., \& Khaliluzzaman, M. (2015). Evaluation of SNR for AWGN, Rayleigh and Rician Fading Channels Under DPSK Modulation Scheme with Constant. International Journal of Wireless Commatications and Mobile Computing $3(1)$ : 7-12.1 https://doi.org/10.11648/j wcmc.20150301.12

Essop, A., \& Xu, H. (2015). Symbol Error Rate of Generalized Selection Combining with Signa Space Diversity in Rayleigh Fading Channels. South African Instistute of Electrica Engineers, 106 (December), 201-211.

Ezea, H. U., Adebusuyi, K., Ofusori, T., \& Ezea, U. R. (2018). An Investigation of the Impacts of Phase Noise on Symbol Error Rate in Quadrature Amplitude Modulation Systems. FUOYE Journal of Engineering and Technology, 3(1):67-7

Kim, C. J., Kim, Y. S., Jeong, G. Y., Mun, J. K., \& Lee, H. J. (1996). SER analysis of QAM with space diversity in Rayleigh fading channels. E T R I Journal, 17 ( 4):2 5 - 35 . https://doi.org/10.4218/etrij.96.0196.0043

Krishna, P., Kumar, T. A., \& Rao, K. K. (2016). M-
QAM BER and SER Analysis of Multipath Fading Channels in Long Term Evolutions LTE ). International Journal of Signal Processing, Image Processing and Pattern Recognition, 9(1):361-368.

Marvin K. Simon, M.-S. A. (2000). Digita Communication over Fading Channels.

Ndujiuba, C. U., Oni, O., \& Ibhaze, A. E. (2015) Comparative Analysis of Digital Modulation Techniques in LTE 4G Systems. Journal of Wireless Networking and Communications, 5(2):60-66.

https://doi.org/10.5923/j.jwnc.20150502.02 Omijeh, B. O., \& Eyo, I. (2016). Comparative Study of Bit Error Rate of Different M-ary Modulation Techniques in AWGN Channel. American Journal of Networks and Communications, 5(5):82-90. https://doi.org/10.11648/j.ajnc.20160505.11 Proakis, J. G., \& Salehi, M. (2008). Digital Communications. McGraw-Hill Higher Education (Vol. 49)

Stewart, B., Barlee, K., Atkinson, D., \& Crockett, L. (2015). Software Defined Radio

Workflow Using MATLAB \& Simulink and the RTL-SDR. Retrieved from All 
Papers/Other/Software_Defined_Radio_usi

ng MATLAB S... -

Software_Defined Radio using MATLAB

Simulink_and_the_RTL-SDR.pdf

Yu, H., \& Wei, G. . (2010). Symbol error probability

of cross QAM in rayleigh fading channels.

IEEE Communications Letters, 14(5), 375-

377. https://doi.org/10.1109/LCOMM

2010.05 .100162 